\title{
Función de dos variables y sucesiones numéricas eventualmente periódicas.
}

Miguel Cerdá Bennassar

Septiembre de 2021

\section{Resumen}

Presento un algoritmo que define una función generadora de secuencias eventualmente periódicas, con los valores del ciclo elegibles y empezadas con cualquier número entero.

\section{Palabras clave}

Secuencias eventualmente periódicas, conjetura de Collatz.

\section{Descripción}

Todas las secuencias generadas con esta función serán eventualmente periódicas, cuyo ciclo podremos elegir asignando un valor a $\mathrm{m}$.

Sean $(k, m) \in \mathbb{Z}$, se define este algoritmo como la función $f(k, m)$, tal que:

$$
f(k, m)= \begin{cases}(k-m) / 2, & \text { si k y m son de la misma paridad. } \\ (3 k+1+m) / 2, & \text { si k y m son de distinta paridad. }\end{cases}
$$

$\operatorname{Dom} f(k, m)=(k+m)>0$.

Para $\forall(k, m) \in \mathbb{Z}$, en un número finito de iteraciones, $k(n)=1-m$.

\section{Propiedades}

1 - Todas las sucesiones generadas serán eventualmente periódicas, de período 2, p1=2-m, $\mathrm{p} 2=1-\mathrm{m}$.

2 - Las secuencias con el mismo valor de k+m, tendrán igual número de elementos y la misma distancia entre ellos, que será igual a la distancia entre los valores de m.

$$
k(n)-k 1(n)=m-m 1 \Longleftrightarrow k+m=k 1+m 1
$$


Ejemplos: $\quad k(37)+m(28)=65$

$$
\begin{aligned}
& k(243)+m(-178)=65 \\
& k(65)+m(0)=65
\end{aligned}
$$

$37,70,21,46,9,28,0,-14,-21,-17,-11,-2,-15,-8$, $-18,-23,-20,-24,-26,-27$.

$243,276,227,252,215,234,206,192,185,189,195$, $204,191,198,188,183,186,182,180,179$.

$65,98,49,74,37,56,28,14,7,11,17,26,13,20,10$, $5,8,4,2,1$

Las tres secuencias tienen 20 elementos.

3 - En todas las secuencias, la diferencia entre el primer elemento ky el último k(n), es igual a $k+m-1$.

$$
k-k(n)=k+m-1
$$

\section{Matrices $M(n)$}

Con los valores de $k$ y de m, formamos una matriz con dos filas e infinitas columnas.

En la primera fila, los números enteros escritos ordenadamente, con los números positivos a la derecha del cero, que representan los posibles valores de $k$.

En la segunda fila, los números enteros escritos ordenadamente, con los números positivos a la izquierda del cero, que representan los valores de $\mathrm{m}$.

Una parte de la matriz con los valores desde - 5 hasta 7 para k y desde 6 hasta - 6 para m:

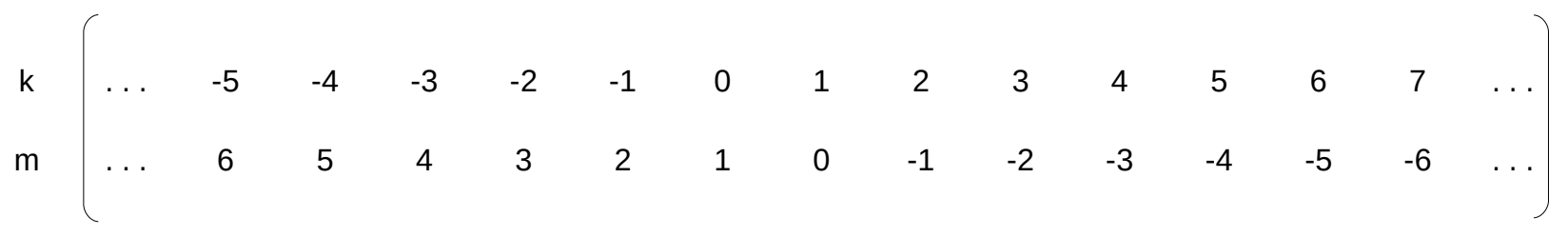

Matriz M(1), en la que $k+m=1$ en cada columna.

Una parte de la matriz con los valores desde 10 hasta 22 para ky desde 6 hasta - 6 para m:

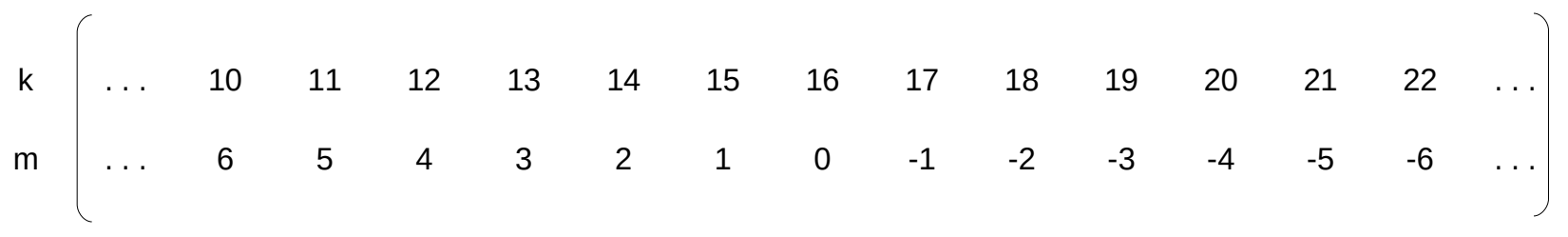

Matriz M(16), porque en cada columna k+m=16.

Los elementos de las dos matrices son los mismos, pero en la matriz M(16) se ha desplazado la primera fila hasta coincidir $\mathrm{k}(16)$ con $\mathrm{m}(0)$, para visualizar que en todas las columnas $\mathrm{k}+\mathrm{m}=16$. 


\section{Conjuntos $C(n)$}

Todas las secuencias generadas con los valores de $k$ y de $m$ de cada columna de la matriz M(n) tienen el mismo número de elementos y hay la misma distancia entre ellos.

Al conjunto de estas secuencias lo llamamos $C(n)$, donde $n=k+m$.

Ejemplo:

Con los valores de las columnas de la matriz M(16), la función generará infinitas secuencias que formarán el conjunto C(16).

$$
C(16)\left\{\begin{array}{ccccc}
\ldots & & & & \\
(10, & 2, & -2, & -4, & -5) ; \\
(11, & 3, & -1, & -3, & -4)_{i} \\
(12, & 4, & 0, & -2, & -3)_{i} \\
(13, & 5, & 1, & -1 & -2) ; \\
(14, & 6, & 2, & 0, & -1)_{i} \\
(15, & 7, & 3, & 1, & 0)_{i} \\
(16, & 8, & 4, & 2, & 1)_{i} \\
(17, & 9, & 5, & 3, & 2)_{i} \\
(18, & 10, & 6, & 4, & 3)_{i} \\
(19, & 11, & 7, & 5, & 4)_{i} \\
(20, & 12, & 8, & 6, & 5)_{i} \\
(21, & 13, & 9, & 7, & 6)_{i} \\
(22, & 14, & 10, & 8, & 7)_{i} \\
\cdots & & & &
\end{array}\right\}
$$

Existen infinitos resultados para $k+m$, que formarán infinitos conjuntos $C(n)$, con las mismas propiedades.

\section{Ejemplos}

Si queremos formar una secuencia que termine en 45 , asignaremos a m el valor de -44 y aplicaremos la siguiente función, de forma iterada, hasta llegar a $k(n)=1-m$ :

$$
f(k, m)= \begin{cases}(k+44) / 2, & \text { si k y m son de la misma paridad. } \\ (3 k-43) / 2, & \text { si k y m son de distinta paridad. }\end{cases}
$$

Porque el dominio de esta función es $(k+m)>0 \rightarrow k \geq 45$.

Secuencia empezada con $k=74, m=-44$ :

$74,59,67,79,97,124,84,64,54,49,52,48,46,45,46,45, \ldots$ 
Secuencia empezada con $\mathrm{k}=12795, \mathrm{~m}=-44$ :

$12795,19171,28735,43081,64600,32322,16183,24253,36358,18201,27280,13662,6853$, $10258,5151,7705,11536,5790,2917,4354,2199,3277,4894,2469,3682,1863,2773,4138$, $2091,3115,4651,6955,10411,15595,23371,35035,52531,78775,118141,177190,88617$, $132904,66474,33259,49867,74779,112147,168199,252277,378394,189219,283807$, 425689, 638512, 319278, 159661, 239470, 119757, 179614, 89829, 134722, 67383, 101053, $151558,75801,113680,56862,28453,42658,21351,32005,47986,24015,36001,53980$, $27012,13528,6786,3415,5101,7630,3837,5734,2889,4312,2178,1111,1645,2446,1245$, $1846,945,1396,720,382,213,298,171,235,331,475,691,1015,1501,2230,1137,1684$, $864,454,249,352,198,121,160,102,73,88,66,55,61,70,57,64,54,49,52,48,46,45,46$, $45, \ldots$

Para todo entero $k \geq 45$, la iteración bajo esta transformación, terminará en 46, 45 .

Si queremos que la secuencia acabe en el número $k(n)=-100$, asignaremos a m el valor de 101 y la iteración bajo esta transformación, para todo número entero $k \geq-100$, terminará en -99, 100.

$$
f(k, m)= \begin{cases}(k-101) / 2, & \text { si k y m son de la misma paridad. } \\ (3 k+102) / 2, & \text { si k y m son de distinta paridad. }\end{cases}
$$

Porque el dominio de esta función es $(k+m)>0 \rightarrow k \geq-100$.

Secuencia empezada con $\mathrm{k}=21, \mathrm{~m}=101$ :

$21,-40,-9,-55,-78,-66,-48,-21,-61,-81,-91,-96,-93,-97,-99,-100,-99,-100, \ldots$

Secuencia empezada con $k=0, m=101$ :

$0,51,-25,-63,-82,-72,-57,-79,-90,-84,-75,-88,-81,-91,-96,-93,-97,-99,-100,-99,-$ $100, \ldots$

\section{Conclusión}

Cualquier número entero $k \in \mathbb{Z}$ del dominio, sometido a la transformación de la función de manera iterada, acabará siempre en $\mathrm{k}(\mathrm{n})=1-\mathrm{m}$.

Con esta función podemos determinar el número entero al que llegará cada secuencia, después de un número finito de iteraciones, en función del valor que asignemos a $m \in \mathbb{Z}$, del dominio.

La conjetura de Collatz se cumplirá para todo valor de $k$, porque en todos los conjuntos $C(n)$ existe una secuencia generada con el valor de $\mathrm{m}=0$ que acabará en $\mathrm{k}(\mathrm{n})=1-\mathrm{m}$, o sea 1 . 
Calculador online de la función, generador de sucesiones: www.riodena.es

Miguel Cerdá Bennassar.

6 de Septiembre de 2021

dosena@riodena.com 